\title{
COMPUTING THE TABLE OF MARKS OF A FINITE GROUP
}

\author{
LIAM NAUGHTON
}

This is an abstract of the $\mathrm{PhD}$ thesis Computing the Table of Marks of a Finite Group written by Liam Naughton under the supervision of Goetz Pfeiffer at the School of Mathematics, Statistics and Applied Mathematics, NUI, Galway and submitted in September 2010 .

In this thesis we introduce a new method for constructing the table of marks of a finite group $S$ from the table of marks of $A$ a normal subgroup of $S$ of index $p$, a prime. The first step in any such approach is to compute a list of representatives of the conjugacy classes of subgroups of $S$. In this spirit we describe a new algorithm which computes the conjugacy classes of subgroups of $S$ from the conjugacy classes of subgroups of $A$. We then present a series of algorithms which compute the table of marks of $S$ from the table of marks of $A$. Computer programs based on the theory described in this thesis have been used to compute the table of marks of $S_{13}$ from the table of marks of $A_{13}$.

School of iMathematics, Statistics and Applied Mathematics, NUi, GALWAY

E-mail address: liam.naughton@nuigalway.ie

2010 Mathematics Subject Classification. 20B05.

Key words and phrases. Table of Marks, Finite Group.

Received on 1-11-2011. 